\title{
Standards and Practices: A Reflection on Programming for Skill Learning and Evaluation in the First Aid Classroom
}

\author{
Adam Gesicki, Canadian Red Cross
}

Every day across the world, learners are exposed to a number of opportunities to learn the skills of first aid, including by way of facilitator-led programming, digital apps, video clips on social media sites, or advertising in television commercials or on billboards. To ensure skill learning is effective over time and repeated exposures, being able to present the learner a consistent message regardless of medium - is an important goal to consider. From the perspective of training organizations with facilitator-based delivery systems, this translates to the need for consistency between instructors to ensure a "common language" is used for skill teaching and evaluation. However, the delivery of first aid is never performed in de-contextualized spaces or in its idealized forms: the provision of first aid is always muddled with factors relating to the first aider, the ill/injured person, and the environment. It is these factors that often force first aiders to adopt and modify the techniques they use to meet their needs, requiring a differentiation - as opposed to standardization - of skill performance. The aim of this paper is to open the conversation on this "middle ground" between these two distinct and important mandates, and to provide a perspective on how the first aid community may want to reconcile these seemingly divergent views.

\section{Perspective of the Learner}

Given that the learner is at the core of first aid learning, it is helpful to reflect on the various ways in which skill acquisition, and its subsequent evaluation, may be perceived by the learner. Consider Table 1: one side features viewpoints learners may hold that support a single "ideal" model for a skill; the other presents learner viewpoints that support multiple approaches in performing a skill.

Although the perspectives shown in the table are hypothetical, it is meant to highlight that the experience of the learner sits with expectations in both realms. Each perspective highlights a valid position, requiring sponsors of first aid learning to appropriately balance both in the delivery of their learning interventions.

\section{Perspectives of Training Organizations}

To-date, training organizations have navigated this space in very different ways. As an example, some organizations differentiate between "standards" vs. "practices". Take the following example, as used by one training organization:

Standards are documented in the skills sheets and are evidence-based, required procedures. Practices are adaptations of standards that users have adopted for a variety of reasons (they see someone else doing it, its perceived as being easier or faster than the standard, they think it is more effective, etc.). Instructors need to be able to distinguish between evidence-supported clinical treatments (standards) and practices employed by learners. Certification requires that participants perform all skills according to the standards outlined in our programs and according to the course's skills sheets.

(CRC, 2018, p. 109)

However, differentiating between a practice and a standard can become easily tangled. Figure 1 shows a 
Table 1. A table illustrating the various perspectives learners may share in support of a single, unified approach for a skill; versus multiple techniques being presented for the same skill.

\begin{tabular}{|c|c|}
\hline Single Model for a Skill & Multiple Models for a Skill \\
\hline $\begin{array}{l}\text { - When I am exposed to the same skill from } \\
\text { different learning mediums over time (such } \\
\text { as facilitator-based, advertisements, or } \\
\text { apps), I should be able to recognize the skill } \\
\text { as the same. This helps me confirm my } \\
\text { existing knowledge, as opposed to making } \\
\text { me feel like I need to "unlearn and relearn" } \\
\text { the same skill. Any differences should be } \\
\text { explainable (e.g., additional science to } \\
\text { support another way of performing a skill, } \\
\text { an easier method was found, etc.). } \\
\text { When the learning intervention I } \\
\text { participate in uses multiple mediums as } \\
\text { part of the same intervention, the skills } \\
\text { should be similar in between all mediums. } \\
\text { For example, a facilitator's demonstration } \\
\text { of skills should match how the skill is } \\
\text { portrayed in the course's materials, or the } \\
\text { instructions demonstrated on an app } \\
\text { should match the type of feedback } \\
\text { provided to me as part of using a feedback } \\
\text { device for autonomous learning. } \\
\text { If a particular medium presented a } \\
\text { multitude of options to perform a skill, it } \\
\text { would become an unwieldy resource. } \\
\text { When I perform first aid in an actual } \\
\text { situation, any person that joins me should } \\
\text { be able to recognize the skill I am } \\
\text { performing and be able to assist me. The } \\
\text { same holds true if I were to witness } \\
\text { someone else doing first aid. }\end{array}$ & $\begin{array}{l}\text { - I may have physical differences as } \\
\text { compared to the person performing the } \\
\text { skill for me to model. However, I can find a } \\
\text { way that works for me that is just as } \\
\text { effective at delivering the same outcome. } \\
\text { - If I find a technique hard to perform, for } \\
\text { whatever reason, I would like the medium } \\
\text { to be able to guide and suggest alternatives } \\
\text { that might help me reach the same final } \\
\text { goal. This might be through facilitator } \\
\text { feedback, or additional options available for } \\
\text { me to select in an app. } \\
\text { Real life may not resemble the classroom. I } \\
\text { want to be given the tools so that I can } \\
\text { adapt the skill for what I may be faced with } \\
\text { outside of the classroom. } \\
\text { I don't want to spend time in the learning } \\
\text { space obsessing over details that feel } \\
\text { "stylistic" and are not supported through } \\
\text { evidence, only with the aim of embodying a } \\
\text { single correct model. Such "nitpicking" } \\
\text { discourages me from learning. } \\
\text { Less emphasis on the details, and more } \\
\text { emphasis on the broader picture, will help } \\
\text { me feel more confident when trying to } \\
\text { deliver first aid when the time comes. I } \\
\text { won't feel stuck in trying to remember all } \\
\text { the details that aren't instrumental to the } \\
\text { delivery of the skill. }\end{array}$ \\
\hline
\end{tabular}

part of a skills sheet used by the same organization as it relates to the responsive choking infant. One can notice that the technique used for the back blows does not specify how they are to be done (Step 2 in Figure 1); however, two fingers are specifically indicated for chest compressions (Step 4 in Figure 1). The compression of

2. Deliver 5 firm back blows between the shoulder blades.

3. If the object does not come out, flip the baby face up, ensuring you support the head.

Figure $1 A$ snippet from a document detailing the steps required for a learner to demonstrate for the responsive choking infant, as required for successful certification (CRC, 2017a). the chest, could of course, be achieved in any number of ways. Such resources may disempower facilitators in correctly disentangling a standard from a practice.

Some work has already occurred on an international stage, recognizing the diversity that can exist in the practices used to achieve the same goal. As one example, leg-foot CPR 
was highlighted in the 2005 Guidelines as an opportunity to deliver compressions of the same depth and rate as compressions delivered by hand (ILCOR, 2005). Even though the technique was validated for use in achieving the same goal, it has not been universally presented to facilitators of learning as an "approved" alternative (e.g., CRC, 2017b), nor is embedded universally within first aid apps (e.g., CRC, 2019). This technique is especially relevant in cases where learners may not be able to use the traditional two-handed technique (e.g., in the case of a first aider having had a bilateral hand amputation); but, given an alternative technique, should be afforded the opportunity to successfully complete a particular learning intervention (e.g., certification) and apply the skill in real life.

Given the value of multiple practices, contrasted with the drive to have a unified perspective on a particular skill, the following definitions are suggested:

- a standard is to be focused on the expected outcome of performing a skill, which is associated with some clinical benefit;

- a practice is one of the many methods that can be used in achieving the expected outcomes (i.e., standard); and,

- a default practice is the practice that has been selected as the primary practice to demonstrate in learner materials and by facilitators of first aid learning. The selection is done with the understanding that it is simply one of the many practices, and that alternative practices may equally achieve the expected standard of the skill.

\section{Linking to Existing Theory}

The proposed definitions bear some analogous links with the Utstein Formula for Survival (Søreide et al., 2013). The Formula is meant to highlight how survivability is influenced by three key factors: medical science, educational efficiency, and local

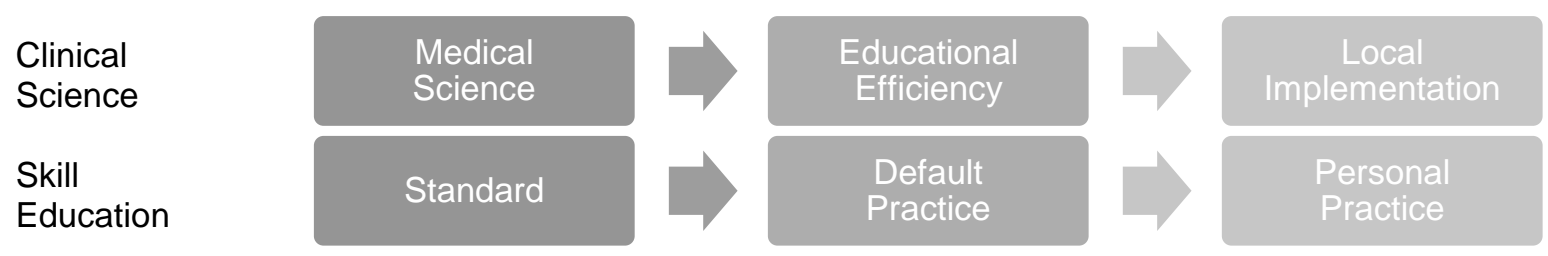

Figure 2: A theoretical association between the Utstein Formula (Søreide et al., 2013, p. 1488) and the proposed definitions. implementation. Although it was created in the specific context of cardiac arrest, it has certainly found an application within the realm of first aid (IFRC, 2016).

Not only does the formula provide an approach to the defining of clinical science, but also connects to the concepts proposed earlier:

- Medical science drives the basis of the skill. In the terminology used in this paper, it is the science that is responsible for building the standard of a skill.

- Educational efficiency captures the drive and necessity to select a single practice to present to learners and is the primary driver behind the factors highlighted in Table 1's left column (single model for a skill). This can be termed the default practice; as in, the practice that is initially taught and presented to learners.

- Ultimately, the highest form of local implementation occurs when a learning medium can customize the skill based on a learner's needs (perceived or observed), and the learner is empowered to customize the skill based on their own needs and that of each situation. These factors may be known in advance of providing care or be determined only at the point of providing care. Local implementation therefore represents a first aider's personal practice.

\section{Application of the Model to Research and Program Development}

Given the definition of these terms, it helps to consider how this framework may be practically applied to the research and program development cycle. When an investigation of a technique is initially performed, researchers are urged on being as descriptive as possible on how the technique was performed in the study. This is done with the aim of enabling reproducibility. In an ideal world, if a technique is described in a specific way 
during its trials, the implementation of the skill should resemble that which was documented in the original study. However, this classic research approach does not necessarily support the use of practices. As a result, each new skill slated for inclusion into a course ends up becoming distilled to its core concepts; namely, the proposed mechanism in which a skill makes an impact on clinical outcomes. It is then the distilled version that becomes the standard for a skill.

Once a standard has been developed, a variety of practices would then need to be identified. These practices would, by necessity and definition, include the description of the practice from any original research studies. This step should have researchers and educators, as well as subject matter experts in accommodations and environmental diversity, join forces to consider alternative ways in which the same standard may be delivered. Each of the potential practices should be subsequently evaluated for their effectiveness. Effectiveness, in this case, is purposefully defined broadly. At a minimum, this process should consider:

a) the ability of presenting the practice through a variety of mediums;

b) the possibility for the intended learner to perform the practice, in terms of physical ability and of risk/safety to the first aider;

c) the practice's ability to meet the standard;

d) the palatability of the learner in using the practice (during learning sessions and in real life);

e) the learner's ability to retain the skill over time;

f) the devices or tools that may be required to complete the practice successfully; and,

g) cultural acceptability of the practice being performed.

This evidence does not necessarily come from experimental studies. Much like the current clinical Guidelines, it may need to be based on expert opinion until such time that further evidence can be collected and integrated. The process of evidence collection and weighing would end with consideration of other local implementation factors (such as jurisdictional/local styles), subsequently leading to the determination of which technique should be appointed to become the default practice. As a living process, it would be subject to revision, based on new perspectives and research that may emerge over time.

The extent to which different first aid providers are aware of various practices needs to be explored in terms of the benefits to the learner, balanced with the expense of the process. A more advanced first aider, who may need to work inter-jurisdictionally, may need to be taught the practices used within all jurisdictions they wish to practice in. A first aider working in more varied contexts, such as one trained for the wilderness, requires an additional understanding of practices and their relative advantages, to be able to deliver care in a more diverse set of environments. Facilitators of learning may need to have a more comprehensive understanding of the variety of practices than a lay provider who received a brief learning intervention.

Once the default practice is identified, training organizations are then charged with producing material to support the implementation of the skill. The default practice becomes depicted in learner materials (e.g., apps, manuals, and the pictures/videos that would accompany such materials) and facilitators are verified to be able to demonstrate the default practice. Additionally, resources would need to be provided to rationalize the various practices, as well as provide inspiration for alternative techniques that may suit the personal needs of the learners into their classroom. Any evaluation processes would need to consider the participant's ability to demonstrate the standard, as opposed to the default practice, or any specific practice that may be contained in resource material.

\section{Conclusion}

It is acknowledged that the suggested process adds another layer of complexity to an already elaborate process of revision within first aid. The process of distilling the clinical science into a standard, identifying potential practices, and evaluating the practices for selection as the default one, would ensure that teaching and learning within the classroom is supported at the individual level and the level of the training organization. This process would ensure that the 
learning of first aid skills is accessible to the broadest range of audiences, broadening even further the impact that a first aid-prepared public can have.

\section{References}

Canadian Red Cross Society, The. (2014). Instructor Worksheet: Red Cross Swim Kids 10. Retrieved from http://myrc.redcross.ca (instructor access required).

Canadian Red Cross Society, The. (2017a). Choking Baby: Skills Sheet. Retrieved from http://lms.redcross.ca/boulevard// (instructor access required).

Canadian Red Cross Society, The. (2017b). First Aid \& CPR. Retrieved from https://www.redcross.ca/facpr Canadian Red Cross Society, The. (2018). First Aid Program Standards - July 2018. Retrieved from http://myrc.redcross.ca (instructor access required).

Canadian Red Cross Society, The. (2019). First Aid - Canadian Red Cross (Version 3.1.5) [Mobile application software]. Retrieved from the Google Play store.

International Federation of Red Cross and Red Crescent Societies. (2016). International first aid and resuscitation guidelines 2016. Retrieved from www.ifrc.org/Global/Publications/Health/First-Aid-2016Guidelines EN.pdf

International Liaison Committee on Resuscitation. (2005). Part 2: Adult basic life support. Circulation, 112, III3 - III-16. doi:10.1161/CIRCULATIONAHA.105.166472

Jones, I., Whitfield, R., Colquhoun, M., Chamberlain, D., Vetter, N., Newcombe, R. (2007). At what age can schoolchildren provide effective chest compressions? An observational study from the Heartstart UK schools training program. British Journal of Medicine. doi: 10.1136/bmj.39167.459028.DE

Søreide, E., Morrison, L.J., Hillman, K., Monsieurs, K., Sunde, K., Zideman, D., Eisenberg, M., Sterz, F., Nadkani, V.M., Soar, J., Nolan, J.P. (2013). The formula for survival in resuscitation. Resuscitation, 84, 1487-1493, 2013. 